\title{
Review of: "Selenium Alleviated Oxidative Stress- Mediated Complex Poisoning Mechanism in Lead- Treated Chicken Kidneys: Inflammation, Heat Shock Response, and Autophagy"
}

\author{
Hongjin Lin ${ }^{1}$ \\ 1 Northeast Agricultural University
}

Potential competing interests: The author(s) declared that no potential competing interests exist.

The research paper by Zhiying Miao et al. aims to evaluate the effect of Se and $\mathrm{Pb}$ on chicken kidneys. They show that $\mathrm{Pb}$ exposure changes renal histological structure, triggers inflammation, heat shock proteins, autophagy. And Se alleviated $\mathrm{Pb}$ poisoning via mitigating oxidative stress in the chicken kidney. Despite an interesting subject, there are many improvements to be made to the manuscript.

Question 1: The language used throughout the paper needs to be checked. Such as “...Although laws and regulations has been implement to control Pb pollution..." in Introduction, "has been implement" should be changed which shows that the author needs to take extra care before submission.

Question 2: The material and methods are too brief and the techniques used are better described.

Question 3: What is the basis of chosen concentrations of Se and $\mathrm{Pb}$ ?

Question 4: In Material and methods - Ultrastructure, please present the PBS composition (even if it seems obvious).

Question 5: What are the exact references of the kits for the measurement of antioxidant activities?

Question 6: The abbreviation list was necessary.

Question 7: In Material and methods - Animal model, what "W" mentioned means? It should be explained.

Question 8: In Results - T-AOC, GST, and CAT activities and H2O2 content in chicken kidneys, its results should be showed in Figure 2 but you wrote Figure 3, please correct it.

Question 9: In Discussion, “...In the present study, we observed high expression of HSPs (HSP27, HSP40, HSP60, HSP70, and HSP90) mRAN and protein caused by...", "mRAN" should be replaced by "mRNA". A careful re-submission after these corrections would result in a commendable publication. 